Military Technical College Kobry El-Kobbah, Cairo, Egypt

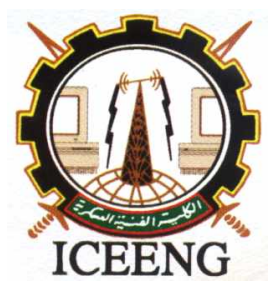

\author{
$6^{\text {th }}$ International Conference \\ on Electrical Engineering \\ ICEENG 2008
}

\title{
Investigation of high mobility two-dimensional electron gas in AlGaN/GaN heterostructures grown by hydride vapor phase epitaxy (HVPE)
}

By

Ashraf Uddin*

Rabah W. Aldhaheri*

Yi Qu**

\section{Abstract:}

Two-inch-diameter sapphire (0001) substrates were used for the growth of AlGaN/GaN structure by hydride vapor phase epitaxy (HVPE). The two-dimensional electron gas (2DEG) structure consisted of a $2 \mu \mathrm{m}$ undoped GaN layer substrate, followed by $30 \mathrm{~nm}$ thick $\mathrm{Al}_{0.3} \mathrm{Ga}{ }_{0.7} \mathrm{~N}$ layer. Structural and optical properties of $\mathrm{GaN}$ layer were characterized by high resolution X-ray diffraction, transmission electron microscopy (TEM) and photoluminescence (PL) measurements. TEM images showed the dislocation density in the GaN layer was $\sim 9 \times 10^{9} / \mathrm{cm}^{2}$. The full width at half maximum (FWHM) values of the x-ray rocking curve for GaN (0002) was $0.156 \mathrm{deg}$. PL spectra of our sample exhibited a predominant band-edge emission of the wurzite $\mathrm{GaN}$ epilayer near $3.36 \mathrm{eV}$ with $\mathrm{FWHM}=45 \mathrm{meV}$ at $50 \mathrm{~K}$ and $75 \mathrm{meV}$ at room temperature, respectively. The Hall effect measurements gave a sheet electron concentration of $7.8 \times 10^{12} \mathrm{~cm}^{-2}$ and a mobility of $850 \mathrm{~cm}^{2} / \mathrm{Vs}$ at $300 \mathrm{~K}$. The experimental data indicated that the HVPE grown GaN layers exhibited superior quality compared to $\mathrm{GaN}$ layers grown by metal organic chemical vapor deposition in prior art literatures.

Keywords: hydride vapor phase epitaxy, GaN, two-dimensional electron gas, heterostructure

* Department of Electrical and Computer Engineering, King Abdulaziz University, PO Box 80204, Jeddah 21589, Saudi Arabia; Email: Uddin98@ hotmail.com

** National Key Laboratory on High Power Semiconductor Lasers Changchun University of Science and Technology No. 7083, Weixing Road, Changchun, P. R. China, 130022 


\section{Introduction:}

$\mathrm{AlGaN} / \mathrm{GaN}$ heterostructures have recently attracted much attention due to the potential application in high-power, high-temperature microelectronic devices. Due to the lack of a natural substrate, most $\mathrm{GaN}$ devices are made from multiple epitaxial layers grown on either sapphire or SiC. In particular, enormous progress has been achieved in the development of $\mathrm{AlGaN} / \mathrm{GaN}$ high electron mobility transistors (HEMTs) due to significant advances in epitaxial growth and processing.[1-2] The large band offset and strong piezoelectric effect in this material system have been shown to induce a high sheet density two-dimensional electron gas (2DEG) with an enhance electron mobility. The GaN-based optoelectronics and microelectronics devices are generally grown by using metal organic chemical vapor deposition (MOCVD) and by molecular beam epitaxy (MBE). Typical values for the room-temperature electron mobility in both MBE and MOCVD grown AlGaN/GaN HEMTs lie in the range of $1000-1600 \mathrm{~cm}^{2} / \mathrm{Vs}$ while the electron sheet density varies from $1 \times 10^{13}$ to $1.5 \times 10^{13} \mathrm{~cm}^{-2}$.[3] On the other hand, hydride vapor phase epitaxy (HVPE) is one of the earliest growth methods employed for GaN [4]. The HVPE technology has been developed to grow thick and low-defect GaN epitaxial layers. Such thick films are difficult to be grown by MBE and MOCVD due to their lower growth rate. Moreover, in HVPE deposition system, the $\mathrm{Ga}$ is usually transported to the growth surface as a volatile chlorine compound, while $\mathrm{NH}_{3}$ is used as the N-precursor. Carbon-free precursors are used in HVPE system and thus this technique could provide high purity and high quality GaN through careful control of the growth process.[4] This enhances the capability of HVPE technology for high-quality nitride-based semiconductor device. Recently, $\mathrm{AlGaN} / \mathrm{GaN}$ high electron mobility transistors (HEMT) grown by HVPE on sapphire substrates were reported [5,6]. The HEMT properties are comparable with the more commonly used MBE and MOCVD grown devices. Now it is desirable to explore the potential of HVPE to grow GaN based optoelelctronics. The HVPE growth system has several advantages over MOCVD and MBE systems such as lower growth costs, higher growth rates, and the demonstrated capability to grow films to thicknesses of tens to hundreds of micrometers. As a result, it is suggested that HVPE could provide high purity and high quality GaN through careful control of the growth process.[7]

In this paper, we report $\mathrm{AlGaN} / \mathrm{GaN}$ 2DEG grown by HVPE on sapphire substrates and the properties are comparable with the more commonly used MBE and MOCVD. Structural and optical properties of GaN layer were characterized by high resolution Xray diffraction, transmission electron microscopy (TEM) and photoluminescence (PL) measurements. The Hall effects measurements gave a sheet electron concentration of $7.8 \times 10^{12} \mathrm{~cm}^{-2}$ and a mobility of $850 \mathrm{~cm}^{2} / \mathrm{Vs}$ at $300 \mathrm{~K}$. The experimental data indicated that the HVPE grown GaN layers exhibited superior quality compared to GaN layers grown by metal organic chemical vapor deposition in prior art literatures. 


\section{Experimental procedure:}

Hydride vapor phase epitaxy method was used to grow GaN layer on two-inch-diameter Sapphire substrates. The HVPE growth system was equipped with a quartz horizontal reactor under atmospheric pressure. $\mathrm{HCl}$ and $\mathrm{NH}_{3}$ were used as precursors and argon was used as a carrier gas. In HVPE reactor, $\mathrm{HCl}$ gas reacted with liquid $\mathrm{Ga}$ to form $\mathrm{GaCl}$ gas, which was transported to the growth zone of the reactor and reacted with $\mathrm{NH}_{3}$, resulting in GaN deposition on sapphire substrate. The HVPE growth machine was equipped with an atmospheric pressure quartz horizontal hot-wall reactor and two-zone resistively heated furnace. The reactor consisted of the main quartz tube, six inlet quartz gas tubes for metal sources, and pedestal for substrates. This setup was equipped with multi-channel gas distribution system with high accuracy mass flow controllers to control $\mathrm{HCl}$ flowes through source channels (Al, $\mathrm{Ga}$, In). In growth processes, Ar was used as carrier gas and ammonia was used as the nitrogen source. Boats containing metallic $\mathrm{Ga}(6 \mathrm{~N})$ and $\mathrm{Al}(5 \mathrm{~N})$ were used as $\mathrm{Ga}$ for $\mathrm{Al}$ sources. Growth rates for $\mathrm{AlGaN}$ and $\mathrm{GaN}$ layers were about $20 \mathrm{~nm} / \mathrm{min}$, and $40 \mathrm{~nm} / \mathrm{min}$, respectively. The structure of the HVPE sample was $2 \mu \mathrm{m}$ undoped $\mathrm{GaN}$ layer, followed by $30 \mathrm{~nm}$ thick $\mathrm{Al}_{0.3} \mathrm{Ga}_{0.7} \mathrm{~N}$ layer. The structure is shown in Fig.1. The growth temperature ranged from 1020 to $1070{ }^{\circ} \mathrm{C}$. High resolution X-ray diffraction (HRXRD) spectra were measured using a Philips X'pert diffractometer with a $\mathrm{Cu} \mathrm{K}_{\alpha}$ radiation source $(1.54 \AA)$ to evaluate the crystal quality and to characterize the phases in the film. Transmission electron microscopy (TEM) investigation was carried out on JEOL operating at $200 \mathrm{kV}$ to examine the microscopic structure and threading dislocation in our sample. Crosssectional and plan view TEM samples were prepared by the standard mechanical polishing and ion-thinning technique to achieve electron transparency. The photoluminescence (PL) measurements were performed at both low and room temperature (RT). The excitation source for PL was a He-Cd laser of a wavelength at $325 \mathrm{~nm}$ with a maximum power of $50 \mathrm{~mW}$. The 2DEG mobility was characterized using conventional van-der Pauw cloverleaf Hall measurement techniques. For the measurement, samples of $5 \times 5 \mathrm{~mm}^{2}$ size were cut from the wafer and Ohmic metal contacts were deposited. The Hall effects measurements were done at $300 \mathrm{~K}$

\section{$\underline{\text { 3. Experimental results }}$}

In the $\theta-2 \theta$ x-ray diffraction pattern (not shown), a narrow and intense GaN (0002) reflection was observed only at $2 \theta=34.6^{\circ}$, indicating that the $\mathrm{GaN}$ has a single phase of wurzite structure. High resolution X-ray diffraction (HRXRD) was measured using a Rigaku diffractometer with a $\mathrm{Cu} \mathrm{K}_{\alpha}$ radiation source (1.54 $\AA$ ). Fig. 2 exhibits the 
HRXRD rocking curves ( $\omega$-scans) of (0002) symmetry planes of GaN epilayers. The full width at half maximum (FWHM) values of (0002) diffraction for the GaN epilayer by HVPE was $0.156 \mathrm{deg}$. The rocking curves of symmetric planes are normally responsive to mosaic distortions but insensitive to the pure edge threading dislocations (TDs) because these planes are undistorted by pure edge TDs [8,9]. The (0002) plane rocking curves can be broadened by the screw and mixed TDs. However, the rocking curves of the (10-12) asymmetry plane can be broadened by all types of TDs including pure edge dislocations. The narrow peak of (0002) diffraction suggested that some screw and mixed TDs were reduced by HVPE growth method. It is also noted that the peak position for (0002) plane, has some shift. According to the XRD spectra, the values of the lattice parameters a and c were estimated to be 3.12 and $5.05 \AA$, respectively. The GaN film in c-direction and in-plane was compressed compared with the unstrained wurzite $\mathrm{GaN}(\mathrm{a}=3.189 \AA$, $\mathrm{c}=5.185 \AA)$. The tensile stress existed in the $\mathrm{GaN}$ film. Thus the (0002) plane rocking curves can be broadened only by the screw and mixed threading dislocations.

Figure 3 reveals the typical TEM images and the transmission electron diffraction pattern of GaN film grown. The diffraction pattern in Fig.3a was taken in the interfacial region between $\mathrm{GaN}$ and substrate with the incident electron beam parallel to the [011] direction. This pattern clearly shows that the GaN layer is epitaxially grown on the substrate. Fig. $3 b$ and $3 c$ were obtained near the [2-1-10] axis zone with the diffraction vector of $\mathbf{g}=(0002)$ and (01-10). According to the dislocation invisibility criterion $\mathbf{g} \cdot \mathbf{b}=$ 0 , the dislocations that are visible for $\mathbf{g}=0002$ two-beam conditions are either pure screw or of mixed screw/edge character. The visible dislocations for $\mathbf{g}=01-10$ twobeam conditions are related to pure edge or mixed character. The distribution of TDs gradually decreased from the interface between the epilayer and the substrate to the film free surface. The density of defects becomes much lower above this defect-concentrated buffer region. Most of TDs are perpendicular to the layers/substrate interface although some of TDs are inclined. These dislocations have generally an edge character. At the top surface of GaN layer, the typical dislocation density was $\sim 2-3 \times 10^{9} / \mathrm{cm}^{2}$ and $5-6 \times$ $10^{9} / \mathrm{cm}^{2}$ for mixed TDs or pure screw (Fig. $3 \mathrm{~b}, \mathbf{g}=0002$ ) and pure edge or mixed TDs (Fig. 3c, $\mathbf{g}=11-20$ ) respectively. The total TD density was readily quantified in plan view TEM image shown in Fig. $3 \mathrm{~d}$ and it is $\sim 9 \times 10^{9} / \mathrm{cm}^{2}$ in our sample. This value is comparable to the GaN films grown on sapphire substrate by MOCVD.[8]

For the photoluminescence measurements, the samples were mounted in a cryostat and immersed in pumped liquid He to achieve a low temperature. We observed the strong band-edge emission at both low and room temperature as shown in Fig. 4. The donoracceptor pair emission was not observed. The band-edge emission peak energy is $~ 3.36$ $\mathrm{eV}$ with FWHM of $45 \mathrm{meV}$ at $50 \mathrm{~K}$ and $\sim 3.34 \mathrm{eV}$ with FWHM of $75.0 \mathrm{meV}$ at RT. 
Two very weak yellow emission peaks at $\sim 2.26$ and $\sim 2.35 \mathrm{eV}$ are observed (not shown in Fig. 4). The sample shows slight blue shift at band-edge emission peak. It seems that the GaN grown by HVPE is under some tensile stress state. The FWHM at RT of our sample is much better than the reported results for GaN epilayer grown on silicon [10] and sapphire [11] by HVPE. The resolution of the spectra was $0.025 \mathrm{~nm}$. Although the FWHM at RT is a bit larger than the best results reported by MOCVD results [12], our results from the HVPE method are quite challenging to other methods and thus has high potential for the device applications. Hall effects measurements gave a sheet electron concentration of $7.8 \times 10^{12} \mathrm{~cm}^{-2}$ and a mobility of $850 \mathrm{~cm}^{2} / \mathrm{Vs}$ at $300 \mathrm{~K}$.

Acknowledgments: This work was supported by the Academic Research Council, Ministry of Education, Singapore (ARC 4/02). This work is partially supported by National Natural Sciences Foundation of China under contracts of 60476026 and 60477010, and also by National Key Lab on High Power Semiconductor Laser of China under contract of 03ZS3603 and 04ZS3601. We have also grateful to the College of Engineering, King Abdulaziz University, Jeddah, Saudi Arabia for the financial support.

\section{Figures:}

\begin{tabular}{|c|}
\hline $30 \mathrm{~nm}$ of $\mathrm{Al}_{0.3} \mathrm{Ga}_{0.7} \mathrm{~N}$ \\
\hline $2 \mu \mathrm{m}$ undoped GaN layer \\
\hline Sapphire Substrate \\
\hline
\end{tabular}

Figure 1: The schematic structure of GaN/AlGaN sample grown by HVPE system 


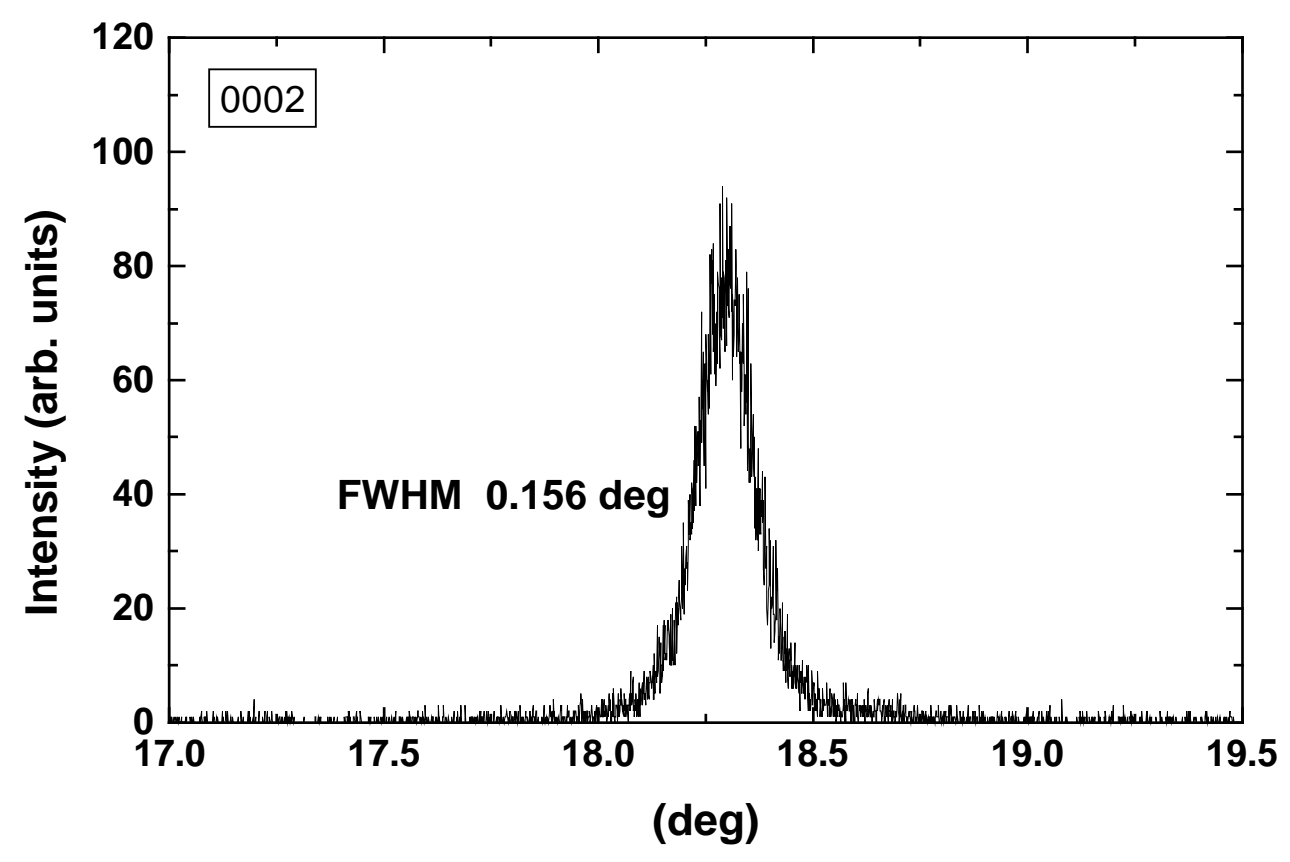

Figure 2: The High resolution X-ray diffraction (HRXRD) rocking curve ( $\omega$-scan) of (0002) symmetry planes of GaN epilayers of 2DEG on sapphire substrate. 


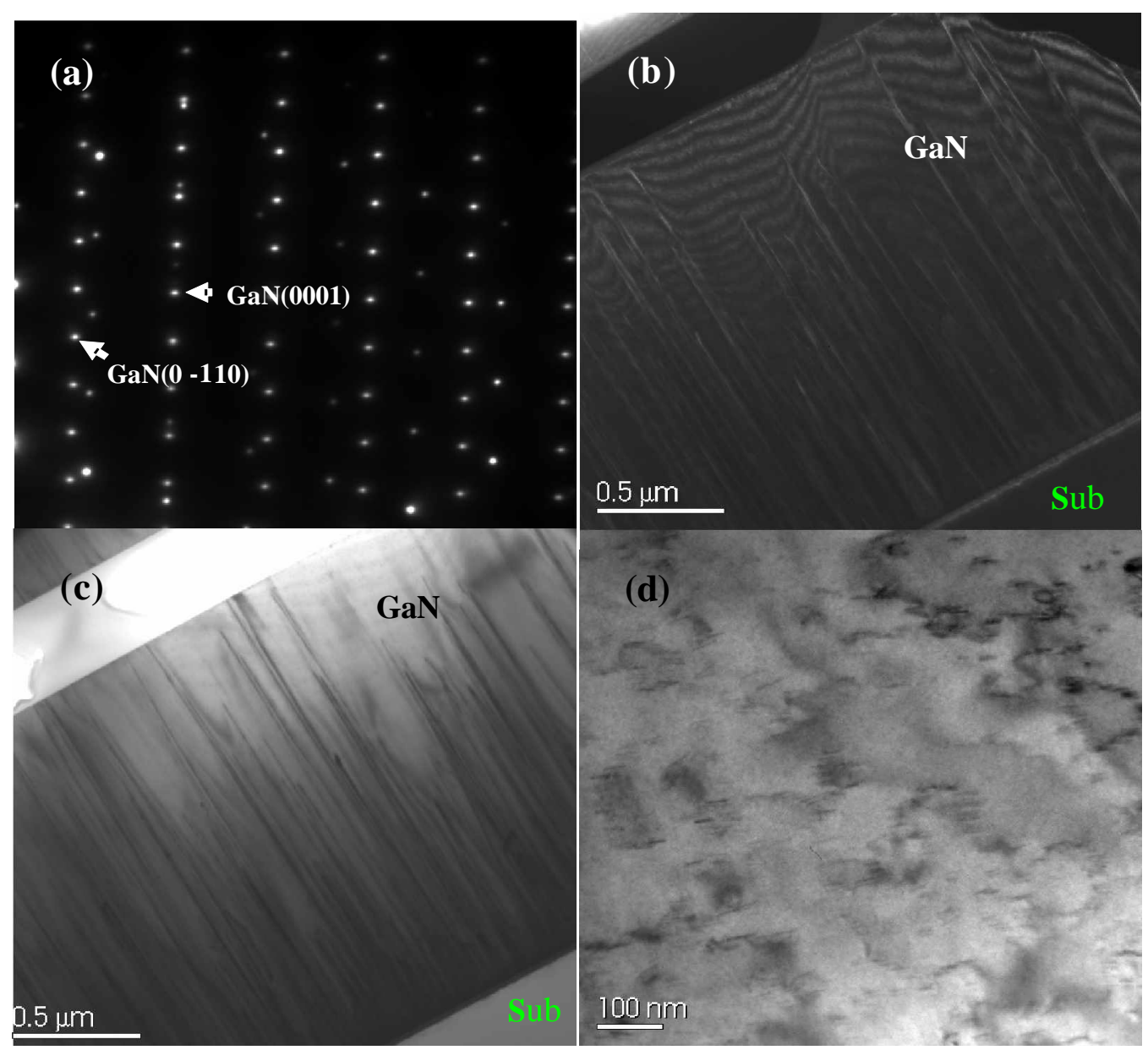

Fig. 3. TEM image and transmission electron diffraction pattern of the GaN epilayer deposited on Sapphire substrate by HVPE. (a) Plan view TEM image showing the dislocation density of the GaN epilayer is $\sim 9 \times 10^{9} / \mathrm{cm}^{2}$. (b) Cross sectional TEM image obtained with reflection vectors $\mathrm{g}=(0002)$. (c) Cross sectional TEM image obtained with reflection vectors $\mathrm{g}=(01-10)$. (d) Transmission electron diffraction pattern of the interfacial region between $\mathrm{GaN}$ and substrate. 


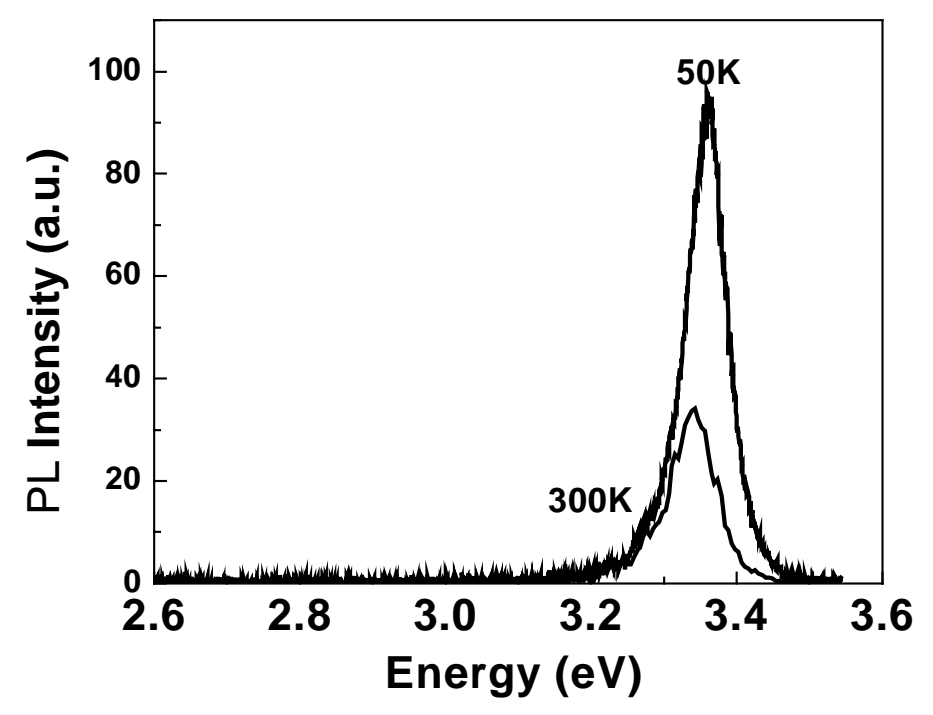

Fig. 4. Photoluminescence spectra of $2 \mathrm{DEG} \mathrm{AlGaN} / \mathrm{GaN}$ structure at $50 \mathrm{~K}$ and room temperature.

\section{Conclusions:}

Two-inch-diameter sapphire (0001) substrates were used for the growth AlGaN/GaN structure by HVPE system. The crystal quality, the threading dislocations and optical properties were characterized by HRXRD, TEM, PL and Hall effect measurements, respectively. The (0002) FWHM values of XRD spectrum was $0.156 \mathrm{deg}$. The total TD density was readily quantified in plan view TEM image and it is $\sim 9 \times 10^{9} / \mathrm{cm}^{2}$ in our sample. This value is comparable to the GaN films grown on sapphire substrate by MOCVD. The PL spectra shows the predominant band-edge emission of the wurtzite GaN epilayer near $3.36 \mathrm{eV}$ with FWHM of $45 \mathrm{meV}$ at $50 \mathrm{~K}$ and $75 \mathrm{meV}$ at RT. Hall effect measurements gave a sheet electron concentration of $7.8 \times 10^{12} \mathrm{~cm}^{-2}$ and a mobility of $850 \mathrm{~cm}^{2} / \mathrm{Vs}$ at $300 \mathrm{~K}$. The GaN layer quality was very high and is comparable to the conventional MOCVD grown layers. Our results suggest that HVPE growth technology has high potential for the fabrication of GaN-based devices on sapphire substrate. 


\section{References:}

[1] S. T. Sheppard, K. Doverspike, W. L. Pribble, S. T. Allen, J. W. Palmour, L. T. Kehias, and T. J. Jenkins, IEEE Electron Device Lett. 20 (1999) 161.

[2] N. X. Nguyen, M. Micovic, W. S. Wong, P. Hashimoto, L. M. McCray, P. Janke, and C. Nguyen, Electron. Lett. 36 (2000) 468.

[3] I. P. Smorchkova, S. Keller, S. Heikman, C. R. Elsass, B. Heying, P. Fini, J. S. Speck, and U. K. Mishra, Applied Physics Lett. 77, (2000) 3998.

[4] T. Detchprohm, K. Hiramatsu, I. Akasaki, Appl. Phys. Lett. 61 (1992) 2688.

[5] M. A. Mastro, D. Tsvetkov, V. Soukhoveev, A. Usikov, V. Dmitriev, B. Luo, F. Ren, K. H. Baik and S. J. Pearton, Solid-State Electron. 47 (2003) 1075.

[6] J. K. Gillespie, R. C. Fitch, N. Moser, T. Jenkins, J. Sewell, D. Via, A. Crespo, A. M. Dabiran, P. P. Chow, A. Osinsky et al., Solid-State Electron. 47 (2003) 1859.

[7] M.A. Mastro, D. Tsvetkov, V. Soukhoveev, A. Usikov, V. Dmitriev, B. Luo, F. Ren, K. H. Baik, and S. J. Pearton, Solid-State Electronics 48 (2004) 179.

[8] K.J. Lee, E.H. Shin, and K.Y. Lim, Appl. Phys. Lett. 85 (2004) 1502.

[9] B. Heying, X.H. Wu, S. Keller, Y. Li, D. Kapolnek, B.P. Keller, S.P. Denbaars, and J.S. Speck, Appl. Phys. Lett. 68 (1996) 643.

[10] P.W. Yu, C.S. Park, S.T. Kim, J. Appl. Phys. 89 (2001) 1692.

[11] E. Oh, S.K. Lee, S.S. Park, K.Y. Lee, I.J. Song, and J.Y. Han, Appl. Phys. Lett. 78 (2001) 273.

[12] A. T. Schremer, J. A. Smart, Y. Wang, O. Ambacher, N. C. MacDonald, and J. R. Shealy, Appl Phys Lett 76, (2000) 736. 